\title{
ON COMPLETE BIORTHOGONAL SYSTEMS
}

\author{
ROBERT M. YOUNG
}

\begin{abstract}
Fundamental to the study of bases in a separable Hilbert space $H$ is the notion of a biorthogonal system. Two sequences $\left\{f_{n}\right\}$ and $\left\{g_{n}\right\}$ of elements from $H$ are said to be biorthogonal if $\left(f_{n}, g_{m}\right)=\delta_{n m}$. A complete sequence that possesses a biorthogonal sequence is called exact. Despite the symmetry of the definition of biorthogonality, simple examples show that $\left\{f_{n}\right\}$ may be exact while $\left\{g_{n}\right\}$ fails to be exact. For sequences of complex exponentials in $L^{2}(-\pi, \pi)$, the situation is dramatically different-if the sequence $\left\{e^{i \lambda_{n} t}\right\}$ is exact, then its biorthogonal sequence is also exact.
\end{abstract}

1. Introduction. Fundamental to the study of bases in a separable Hilbert space $H$ is the notion of a biorthogonal system (see, e.g., [2] and [3] and the references therein).

Definition. Two sequences $\left\{f_{n}\right\}$ and $\left\{g_{n}\right\}$ of elements from $H$ are said to be biorthogonal if $\left(f_{n}, g_{m}\right)=\delta_{n m}$. A sequence that admits a biorthogonal sequence will be called minimal.

It is easy to show that a sequence $\left\{f_{n}\right\}$ is minimal if and only if none of its elements can be approximated by linear combinations of the others. If this is the case, then a biorthogonal sequence will be uniquely determined if and only if $\left\{f_{n}\right\}$ is complete. (This means that the set of all linear combinations $c_{1} f_{1}+\cdots+c_{n} f_{n}$ is dense in $H$, or, equivalently, that the zero vector alone is perpendicular to every $f_{n}$.) A sequence that is both minimal and complete will be called exact.

Despite the symmetry of the definition, it is apparent that for a minimal sequence the property of being complete is not inherited by its biorthogonal sequence. (Cf. if $\left\{f_{n}\right\}$ is a basis, then $\left\{g_{n}\right\}$ is also a basis [4, p. 29].) Indeed, if $\left\{e_{n}\right\}$ is an orthonormal basis for $H$, then $\left\{e_{n}+e_{1}\right\}_{n=2}^{\infty}$ is complete and has $\left\{e_{n}\right\}_{n=2}^{\infty}$ as its biorthogonal sequence. The following generalization is perhaps even more striking.

EXAMPLE. For each infinite-dimensional closed subspace $K$ of $H$, there exists a biorthogonal system $\left\{f_{n}\right\},\left\{g_{n}\right\}$ such that $\left\{f_{n}\right\}$ is complete and the closed subspace spanned by $\left\{g_{n}\right\}$ is $K$. Indeed, let $\left\{e_{n}\right\}$ be an orthonormal basis for $K$ and $B$ an orthonormal basis for $K^{\perp}$. We consider the sequence $\left\{e_{n}+y_{n}\right\}$ where the $y_{n}$ are chosen from $B$ in such a way that each element of $B$ appears as a $y_{n}$ infinitely many times. It is readily shown that $\left\{e_{n}+y_{n}\right\}$ and $\left\{e_{n}\right\}$ are biorthogonal and that the former sequence is complete.

Received by the editors February 2, 1981.

1980 Mathematics Subject Classification. Primary 42C30; Secondary 30D99.

Key words and phrases. Biorthogonal system, exact sequence, Paley-Wiener space. 
The purpose of this note is to show that for minimal sequences of complex exponentials $\left\{e^{i \lambda_{n} t}\right\}$ in $L^{2}(-\pi, \pi)$ the situation is dramatically different-the completeness of such a sequence always ensures the completeness of its biorthogonal sequence.

THEOREM. If the sequence of complex exponentials $\left\{e^{i \lambda_{n} t}\right\}$ is exact in $L^{2}(-\pi, \pi)$, then its biorthogonal sequence is also exact.

2. The Paley-Wiener space. We shall make use of the Paley-Wiener space $P$ consisting of all entire functions of exponential type at most $\pi$ that are squareintegrable on the real axis. It is clear that $P$ is a vector space under pointwise addition and scalar multiplication; it is also an inner product space with respect to the inner product

$$
(f, g)=\int_{-\infty}^{\infty} f(x) \overline{g(x)} d x .
$$

If $f \in P$, then the Paley-Wiener theorem shows that

$$
f(z)=\frac{1}{2 \pi} \int_{-\pi}^{\pi} \phi(t) e^{i z t} d t,
$$

with $\phi \in L^{2}(-\pi, \pi)$. Since the Fourier transform is an isometry, $P$ is a separable Hilbert space, isometrically isomorphic to $L^{2}(-\pi, \pi)$.

Accordingly, problems involving complex exponentials in $L^{2}(-\pi, \pi)$ can be examined via their transform image in $P$. Thus, for example, the sequence $\left\{e^{i \lambda_{n} t}\right\}$ is complete in $L^{2}(-\pi, \pi)$ if and only if $\left\{\lambda_{n}\right\}$ is a set of uniqueness for $P$, i.e., if the relations

$$
f \in P \quad \text { and } f\left(\lambda_{n}\right)=0 \quad(n=1,2,3, \ldots)
$$

imply that $f \equiv 0$. (A detailed account of the Paley-Wiener space and its role in the study of completeness and expansion properties of sets of complex exponentials can be found in [4].)

3. Proof of the theorem. It is convenient to describe the biorthogonal sequence in terms of an entire generating function which vanishes at the $\lambda_{n}$. Let $S$ be the class of entire functions $f(z)$ of exponential type at most $\pi$ such that $f(x) /\left(1+x^{2}\right)^{1 / 2} \in$ $L^{2}(-\infty, \infty)$. The existence of a function $f \in S$, equal to zero at every $\lambda_{n}$, but not identically zero, is necessary and sufficient for $\left\{e^{i \lambda_{n} t}\right\}$ to be minimal $[1$, p. 419]. Notice that $f(z)$ can have no zeros different from the $\lambda_{n}$. Otherwise, additional exponentials could be added to the sequence $\left\{e^{i \lambda_{n} t}\right\}$ without destroying its minimality, and this contradicts its completeness. It follows in the same way that all the zeros of $f(z)$ must be simple, and so $f^{\prime}\left(\lambda_{n}\right)$ is never zero. Furthermore, if $g(z)$ is any other function in $S$, equal to zero at every $\lambda_{n}$, then $g(z)=A f(z)$. For if $\lambda$ is any one of the $\lambda_{n}$, then the function $f(z) / f^{\prime}(\lambda)(z-\lambda)-g(z) / g^{\prime}(\lambda)(z-\lambda)$ belongs to $P$ (provided $g \neq 0$ ), equals zero at every $\lambda_{n}$, and so must be identically zero.

By virtue of the Paley-Wiener theorem, we can write

$$
\frac{f(z)}{f^{\prime}\left(\lambda_{n}\right)\left(z-\lambda_{n}\right)}=\frac{1}{2 \pi} \int_{-\pi}^{\pi} g_{n}(t) e^{i z t} d t,
$$


with $g_{n} \in L^{2}(-\pi, \pi)$. Thus the sequence $\left\{\overline{g_{n}(t)}\right\}$ is biorthogonal to $\left\{e^{i \lambda_{n} t}\right\}$. To prove it is complete, suppose that for some function $h \in L^{2}(-\pi, \pi)$,

$$
\int_{-\pi}^{\pi} h(t) g_{n}(t) d t=0 \quad(n=1,2,3, \ldots) .
$$

It is to be shown that $h(t)=0$ almost everywhere. Replacing $h(t)$ by its Fourier series $h(t)=\Sigma_{-\infty}^{\infty} a_{k} e^{i k t}\left(\Sigma_{-\infty}^{\infty}\left|a_{k}\right|^{2}<\infty\right)$, and then integrating term-by-term, we find

$$
\sum_{-\infty}^{\infty} a_{k} \int_{-\pi}^{\pi} g_{n}(t) e^{i k t} d t=0 \quad(n=1,2,3, \ldots) .
$$

Let us suppose first that no $\lambda_{n}$ is an integer. Then (1) and (2) show that

$$
\sum_{-\infty}^{\infty} a_{k} \frac{f(k)}{k-\lambda_{n}}=0 \quad(n=1,2,3, \ldots) .
$$

Put $c_{k}=a_{k} f(k) / k(k \neq 0)$. Since $f(k) /\left(k-\lambda_{1}\right)$ are the Fourier coefficients of a function in $L^{2}(-\pi, \pi),\{f(k) / k\} \in l^{2}$, and hence $\Sigma\left|c_{k}\right|<\infty$. This permits us to write $c=\Sigma c_{k}$. Since

$$
a_{k} \frac{f(k)}{k-\lambda_{n}}=c_{k} \frac{k}{k-\lambda_{n}}=c_{k}\left(1+\frac{\lambda_{n}}{k-\lambda_{n}}\right) \quad(k \neq 0),
$$

(3) becomes

$$
-\frac{a_{0} f(0)}{\lambda_{n}}+c+\lambda_{n} \sum_{k \neq 0} \frac{c_{k}}{k-\lambda_{n}}=0 \quad(n=1,2,3, \ldots) .
$$

We define a function $g(z)$ by writing

$$
g(z)=-a_{0} f(0) \frac{\sin \pi z}{z}+c \sin \pi z+z \sin \pi z \sum_{k \neq 0} \frac{c_{k}}{k-z}=g_{1}+g_{2}+g_{3} .
$$

AsSERTION. $g(z)$ belongs to the class $S$. Clearly, $g_{1}$ and $g_{2}$ belong to $S$. Since the set of functions $\{(\sin \pi(z-k)) / \pi(z-k)\}_{-\infty}^{\infty}$ forms an orthonormal basis for $P$ (take the Fourier transform of $e^{i k t}$ ) and $\Sigma\left|c_{k}\right|^{2}<\infty$, it follows that the series

$$
\sum_{k \neq 0} \pi(-1)^{k+1} c_{k} \frac{\sin \pi(z-k)}{\pi(z-k)}=\sin \pi z \sum_{k \neq 0} \frac{c_{k}}{k-z}
$$

converges in the topology of $P$, and hence pointwise, to a function belonging to $P$. Thus $g_{3}$ belongs to $S$. This proves the assertion. Since $g\left(\lambda_{n}\right)=0(n=1,2,3, \ldots)$, we must have $g(z)=A f(z)$.

If $k \neq 0$, then

$$
g(k)=k \pi(-1)^{k+1} c_{k}=\pi(-1)^{k+1} a_{k} f(k) .
$$

If $k=0$, the first term is still equal to the last. Accordingly,

$$
A=\pi(-1)^{k+1} a_{k} \text { for every } k,
$$

since $f(k)$ is never zero (recall that no $\lambda_{n}$ is an integer and $f(z)$ is equal to zero only at the $\lambda_{n}$ ). But $\left\{a_{k}\right\} \in l^{2}$, and therefore (4) is possible only if $a_{k}=0$ for every $k$. 
Thus $h(t)=0$ almost everywhere and the biorthogonal sequence $\left\{\overline{g_{n}(t)}\right\}$ is complete in $L^{2}(-\pi, \pi)$. This proves the theorem under the assumption that no $\lambda_{n}$ is an integer.

In the general case, we can choose a suitable real number $\alpha$ such that $\lambda_{n}+\alpha$ is never an integer. Since multiplication by $e^{i \alpha t}$ is a unitary operator on $L^{2}(-\pi, \pi)$, the sequence $\left\{e^{i\left(\lambda_{n}+\alpha\right) t}\right\}$ is complete and hence its biorthogonal sequence $\left\{e^{i \alpha t} \overline{g_{n}(t)}\right\}$ is also complete. Thus $\left\{\overline{g_{n}(t)}\right\}$ is exact.

REMARK. The examples in $\$ 1$ show that the converse of the theorem is falsethere are incomplete sequences of complex exponentials that admit complete biorthogonal sequences. Whether every such sequence must admit a complete biorthogonal sequence appears to be an open problem.

\section{REFERENCES}

1. B. Ja. Levin, Distribution of zeros of entire functions, Transl. Math. Monographs, vol. 5, Amer. Math. Soc., Providence, R. I., 1964.

2. J. Lindenstrauss and L. Tzafriri, Classical Banach spaces. I, Springer-Verlag, Berlin and New York, 1977.

3. I. Singer, Bases in Banach spaces. I, Springer-Verlag, Berlin and New York, 1970.

4. R. M. Young, An introduction to nonharmonic Fourier series, Pure and Appl. Math., vol. 93, Academic Press, New York, 1980.

Department of Mathematics, Oberlin College, Oberlin, Oho 44074

Current address: Department of Mathematics, University of California, Los Angeles, California 90024 